\title{
Modeling the Impact of COVID-19 Vaccination in Lebanon: A Call to Speed-Up Vaccine Roll Out
}

\author{
Ghina R. Mumtaz 1,2,*(D), Fadi El-Jardali ${ }^{3,4,5}$, Mathilda Jabbour ${ }^{3,4}$, Aya Harb ${ }^{3,4}$, Laith J. Abu-Raddad ${ }^{6,7} \mathbb{D}$ \\ and Monia Makhoul 6,7,*(i) \\ 1 Department of Epidemiology and Population Health, Faculty of Health Sciences, \\ American University of Beirut, Beirut 1107 2020, Lebanon \\ 2 Center for Infectious Diseases Research, Faculty of Medicine, American University of Beirut, \\ Beirut 1107 2020, Lebanon \\ 3 Department of Health Management and Policy, Faculty of Health Sciences, American University of Beirut, \\ Beirut 1107 2020, Lebanon; fe08@aub.edu.lb (F.E.-J.); mj93@aub.edu.lb (M.J.); ah291@aub.edu.lb (A.H.) \\ 4 Knowledge to Policy (K2P) Center, Faculty of Health Sciences, American University of Beirut, \\ Beirut 1107 2020, Lebanon \\ 5 Department of Health Research Methods, Evidence, and Impact, McMaster University, \\ Hamilton, ON L8N 3Z5, Canada \\ 6 Infectious Disease Epidemiology Group, Weill Cornell Medical College-Qatar, Cornell University, \\ Qatar Foundation-Education City, Doha 24144, Qatar; lja2002@qatar-med.cornell.edu \\ 7 Department of Healthcare Policy and Research, Weill Cornell Medical College, Cornell University, \\ New York, NY 10022, USA \\ * Correspondence: gm15@aub.edu.lb (G.R.M.); mom2039@qatar-med.cornell.edu (M.M.)
}

Citation: Mumtaz, G.R.; El-Jardali, F.; Jabbour, M.; Harb, A.; Abu-Raddad, L.J.; Makhoul, M. Modeling the Impact of COVID-19 Vaccination in Lebanon: A Call to Speed-Up Vaccine Roll Out. Vaccines 2021, 9, 697. https://doi.org/10.3390/

vaccines 9070697

Academic Editor: Ralph A. Tripp

Received: 27 May 2021

Accepted: 21 June 2021

Published: 25 June 2021

Publisher's Note: MDPI stays neutral with regard to jurisdictional claims in published maps and institutional affiliations.

Copyright: (c) 2021 by the authors Licensee MDPI, Basel, Switzerland. This article is an open access article distributed under the terms and conditions of the Creative Commons Attribution (CC BY) license (https:// creativecommons.org/licenses/by/ $4.0 /)$.
Abstract: Four months into the SARS-CoV-2 vaccination campaign, only $10.7 \%$ of the Lebanese population have received at least one dose, raising serious concerns over the speed of vaccine rollout and its impact in the event of a future surge. Using mathematical modeling, we assessed the short-term impact of various vaccine roll-out scenarios on SARS-CoV-2 epidemic course in Lebanon. At current population immunity levels, estimated by the model at $40 \%$ on 15 April 2021, a large epidemic wave is predicted if all social distancing restrictions are gradually eased and variants of concern are introduced. Reaching $80 \%$ vaccine coverage by the end of 2021 will flatten the epidemic curve and will result in a $37 \%$ and $34 \%$ decrease in the peak daily numbers of severe/critical disease cases and deaths, respectively; while reaching intermediate coverage of $40 \%$ will result in only a $10-11 \%$ decrease in each. Reaching $80 \%$ vaccine coverage by August would prevent twice as many severe/critical disease cases and deaths than if it were reached by December. Easing restrictions over a longer duration resulted in more favorable vaccination impact. In conclusion, for vaccination to have impact in the short-term, scale-up has to be rapid and reach high coverage (at least $70 \%$ ), while sustaining social distancing measures during roll-out. At current vaccination pace, this is unlikely to be achieved. Concerted efforts need to be made to overcome local challenges and substantially scale up vaccination to avoid a surge that the country, with its multiple crises and limited health-care capacity, is largely unprepared for.

Keywords: SARS-CoV-2; COVID-19; coronavirus; epidemiology; vaccine; mathematical model; Lebanon

\section{Introduction}

Lebanon documented its first severe acute respiratory syndrome coronavirus 2 (SARSCoV-2) case on 21 February 2020. After almost a year of ongoing cycles of imposing and easing restrictions to suppress the epidemic, the country experienced its first large wave in December 2020-January 2021 which stretched its fragmented, under-resourced, and highly privatized health care system beyond capacity. As of 16 June 2021, about 543,000 confirmed cases and 7808 deaths have been documented [1], for a population of 6.8 million inhabitants [2]. 
While the pandemic has severely impacted economies and societies worldwide, it was compounded in Lebanon by multiple crises including an unpreceded devaluation of the local currency, political uprising against the ruling class, and the world's largest non-nuclear explosion that blasted its capital Beirut in August 2020, causing thousands of casualties and destroying several hospitals with Coronavirus Disease 2019 (COVID-19) units. With over one million refugees on its territory, Lebanon also hosts the highest per capita number of refugees worldwide [3], a population highly vulnerable to large SARS-CoV-2 outbreaks [4]. At this stage, mass vaccination with the recently available and highly efficacious COVID-19 vaccines [5-9] brings hope of controlling infection spread and mitigate its profound impact, especially with the emerging 'real-world' evidence of their high effectiveness in reducing both symptomatic and asymptomatic disease [10-13].

On 14 February 2021 the Lebanese Ministry of Public Health launched its vaccination campaign and inoculated its first residents. To date, doses enough to ensure a $73 \%$ coverage of the population have been reserved. However, only a small fraction of these doses has been received so far and, four months into the vaccination campaign, only $10.7 \%$ of Lebanese residents have received at least one dose of the vaccine [14], raising serious concerns over supply and the speed of vaccine roll-out. Also, only $25 \%$ of the population have registered to receive the vaccine on the ministry's official vaccine platform [14]. This is likely due to multiple factors including difficulties accessing and navigating the online registration platform by a population not used to online government services, hesitancy around taking the vaccine due to misinformation propagated through the tightly knit social networks in this small population, and a general lack of trust in the government and the national public health response to the epidemic. The recent marked decline in infections may also provide a false sense of security and dissuade people from taking the vaccine.

With the expected relaxation of social distancing restrictions in the upcoming summer months and with the threat of the potential introduction of more transmissible and likely more severe SARS-CoV-2 variants [15-17], the objective of this study was to forecast the short term (by end of year) impact of COVID-19 vaccination in Lebanon in order to inform policy and the national vaccination strategy. We simulate what the epidemic course would be if all restrictions are gradually eased and if variants of concern are introduced, and assess the impact of different levels of vaccine coverage, different durations for vaccine scale-up, and different schedules for easing social distancing restrictions on SARS-CoV-2 incidence and associated burden of COVID-19 disease and death.

\section{Materials and Methods}

\subsection{Mathematical Model Description}

We adapted an existing mathematical model that investigates the generic populationlevel impact of SARS-CoV-2 vaccination and extended it to be applied to Lebanon [18-20]. This is a deterministic age-structured Susceptible-Exposed-Infectious-Recovered (SEIR) model that stratifies the population into compartments reflecting their vaccination status, age group, infection status, infection stage, and disease stage. The model consists of a set of coupled nonlinear differential equations that depict population movement between the different compartments. An age-mixing matrix allowed a range of assortativeness for contact patterns between individuals in different age groups. Population demography was assumed to be stable given the short-term timeframe of the predictions (one year).

We assumed that the duration of natural immunity (following recovery from a natural infection) and vaccine-acquired immunity is one year [21-25]. We further assumed that waning of immunity follows a gamma distribution where only a small fraction of individuals lose their immunity either much earlier or much later than one year. The model was coded, fitted, and analyzed using MATLAB R2019a [26]. Details of the model structure and equations can be found in the Supplementary Information Text S1A, S1B and Table S1. 


\subsection{Model Parameterization, Fitting, and Application to Lebanon}

The model was parametrized with most recent SARS-CoV-2 epidemiological and natural history data. Model parameters, values, and justifications can be found in the Supplementary Information Text S1C and Table S2. The model was fitted to SARS-CoV-2 case series of infections and deaths in Lebanon [1]. Fitting used a nonlinear least square technique, based on the Nelder-Mead simplex algorithm. Given the large wave that happened near the end of year holidays and informed by unpublished clinical antibody testing reports, we assumed that $20 \%$ of the Lebanese population had already been exposed to the infection by 1 January 2021.

Since the ultimate objective of vaccination is to allow a return of social and economic life back to normalcy, we gradually eased all social and physical distancing restrictions over a period of four months starting 15 April 2021. We assumed a constant SARS-CoV-2 incidence rate between 1 January 2021 and 15 April 2021, to reflect the observed relative stability of the epidemic during this time where an average of 3000 confirmed daily cases were being reported [1]. As restrictions were gradually eased starting 15 April 2021, $R_{0}$ was gradually increased to reach $R_{0}=6$ at the end of the four months, thus accounting for the increased transmissibility in absence of non-therapeutic interventions of the new SARS-CoV-2 variants of concern [15,27-31].

\subsection{Vaccine Characteristics and Scale-Up Scenarios}

In Lebanon, the BNT162b2 mRNA COVID-19 Vaccine (Pfizer-BioNTech, Mainz, Germany) is the main vaccine rolled out and has the largest fraction of the doses reserved, followed more recently by the ChAdOx1 nCoV-19 vaccine (AstraZeneca, Cambridge, UK). We assumed that the vaccine has 'real-world' effectiveness of $80 \%$ at reducing infection $\left(\mathrm{VE}_{\mathrm{S}}=80 \%\right)$, based on recent evidence for the effectiveness of the vaccine in reducing asymptomatic as well as symptomatic infections [10,12,32].

Vaccination was introduced on 14 February 2021 (actual date) and scaled-up at a linear rate to reach the desired coverage by 31 December 2021, as informed by the schedule of incoming vaccine shipments to Lebanon where more doses are expected to become available over time. Two scenarios are presented: (1) $80 \%$ vaccine coverage is reached by the end of the year (target coverage rate), and (2) $40 \%$ vaccine coverage is reached by the end of the year (more realistic scenario given current vaccination roll out). During vaccine roll-out, we assumed that, at each point in time, all individuals in the population have an equal chance of getting vaccinated, including those that had already acquired the infection naturally and recovered from it. Vaccine coverage was defined as the proportion of the population that had received two doses of the vaccine and completed 14 days after the second dose at a given point in time.

\subsection{Measures of Vaccine Impact}

After easing all restrictions over four months and introducing variants of concern starting 15 April 2021, we assessed the population-level impact of SARS-CoV-2 vaccination by quantifying and comparing the incident number of infections, severe/critical disease cases, and deaths for the scenarios of (1) no vaccination, (2) vaccine introduced on 14 February 2021 and $80 \%$ vaccine coverage is reached by the end of the year, and (3) vaccine introduced on 14 February 2021 and 40\% vaccine coverage is reached by the end of the year. We used the World Health Organization (WHO) guidelines for classifying infection severity [33] and determining COVID-related death [34].

We further compared the cumulative number of averted severe/critical disease cases and deaths from 14 February 2021 (date of vaccine introduction) to 31 December 2021 for a range of vaccine coverage attained by end of year, for different durations of vaccine scale-up to reach the target $80 \%$ coverage (thus assessing impact of the speed of rollout), and for different durations over which restrictions are eased while reaching the target $80 \%$ coverage by end of year 2021. The impact of vaccine coverage and scale-up duration is assessed regardless of the logistical and structural factors that may affect these measures 
such as vaccine hesitancy, public awareness levels, and low vaccine supply, among others. We did not assess the impact of vaccine prioritization by age as it was investigated in earlier studies [18,35].

\subsection{Uncertainty Analysis}

Uncertainty analysis was conducted to investigate the impact of uncertainty around our assumed transmissibility of the new variants of concern introduced on 15 April 2021 on the model's predictions of the COVID-19 severe/critical disease incidence for the different scenarios. For a total of 500 simulation runs, we used, in each run, Latin Hypercube sampling $[36,37]$ to select an $R_{0}$ within $\pm 30 \%$ of the assumed baseline value of $R_{0}=6$. The resulting distribution for the predicted number of incident and cumulative severe/critical disease cases across the 500 runs was used to calculate the predicted mean and 95\% uncertainty intervals around these two estimates.

\section{Results}

Based on the model assumptions, we estimated that $44 \%$ of the Lebanese population have acquired either natural or vaccine-induced immunity to SARS-CoV-2 by 15 April 2021. With this herd immunity level in the population, gradually lifting all social distancing restrictions by mid-August 2021 and introducing more transmissible variants of the virus will result in a large new epidemic wave over the summer months (Figure 1). While it did not fully prevent the new surge, the $80 \%$ vaccination coverage flattened the epidemic curve (yellow curve), resulting in a smaller epidemic. Under this scenario, the peak incidence of infections, severe/critical disease cases, and deaths are reduced by $37.1 \%, 36.6 \%$, and $34.2 \%$, respectively, compared with the no vaccination scenario (Figure 1). Vaccination did not fully prevent a new epidemic surge because the current contact rate in the community is not sufficiently low and the population immunity level is not sufficiently high, and because of the higher transmissibility of the new variants.

The $40 \%$ vaccination coverage was not able to noticeably flatten the curve (Figure 1 , orange curve) and hence had a much smaller impact on epidemic course compared with the target $80 \%$ coverage rate. This scenario resulted only in an $11.0 \%, 10.8 \%$, and $10.0 \%$ decrease in the peak incidence of infections, severe/critical cases, and deaths, respectively, compared with the no vaccination scenario (Figure 1 ). Reaching $40 \%$ coverage by the end of the year means that most vaccinations would be administered after the predicted time of the summer surge, which undermined vaccine impact under this scenario.

The cumulative numbers of averted severe/critical disease cases and deaths increased substantially and steadily with higher vaccine coverage at the end of 2021 (Figure 2). For example, reaching $80 \%$ vaccine coverage will result in a three-fold increase in the number of prevented severe/critical cases and deaths compared with $40 \%$ vaccine coverage rate. The gains in averted disease cases and deaths increased sharply as vaccine coverage reaches $70 \%$. Reaching $80 \%$ vaccination coverage by the end of the year will prevent a total of 23,600 cumulative severe/critical cases and 2188 cumulative deaths between 14 February 2021 and 31 December 2021 compared with the no vaccination scenario (Figure 2).

The effectiveness of the vaccine in preventing severe/critical disease and death was substantially enhanced by more rapid scale-up to reach the $80 \%$ target vaccine coverage by the end of the year (Figure 3). For example, reaching $80 \%$ vaccine coverage by August would prevent twice as many severe/critical disease cases and deaths than if it were reached by December (Figure 3).

In all of the above scenarios, we assume that easing of all restrictions would occur over four months starting on 15 April 2021. However, as expected, we found that a longer duration over which restrictions are eased would result in a more favorable impact of vaccination (Figure 4). For example, if easing of restrictions happens over eight months, that is people would still exert some levels of social distancing until December 2021 when the target $80 \%$ vaccine coverage is reached, twice as many deaths would be averted than 
if social contacts are back to normalcy by August 2021 (4175 vs. 2185 averted deaths, respectively) (Figure 4).

The extent of transmissibility of the variants of concern introduced on 15 April 2021 remains uncertain. Analysis factoring in the uncertainty around the assumed $R_{0}$ of these variants indicates that the value of $R_{0}$ will affect the scale of the predicted surge (Figure S1A) and, hence, will affect the cumulative number of averted infections (Figure S1B), with higher $\mathrm{R}_{0}$ leading to more averted infections. However, the beneficial vaccine impact relative to the no vaccination scenario remains largely unchanged.

(A)

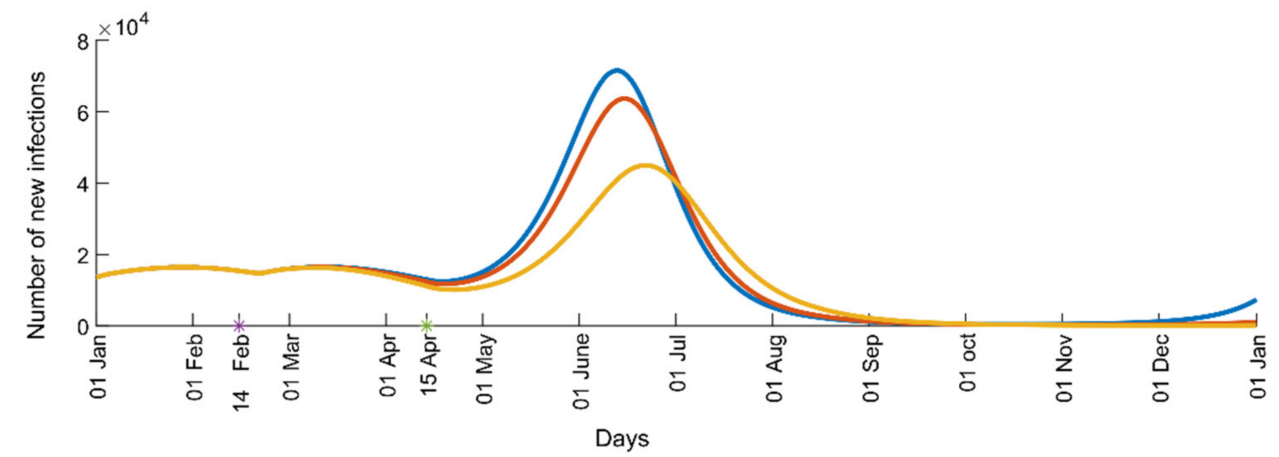

(B)
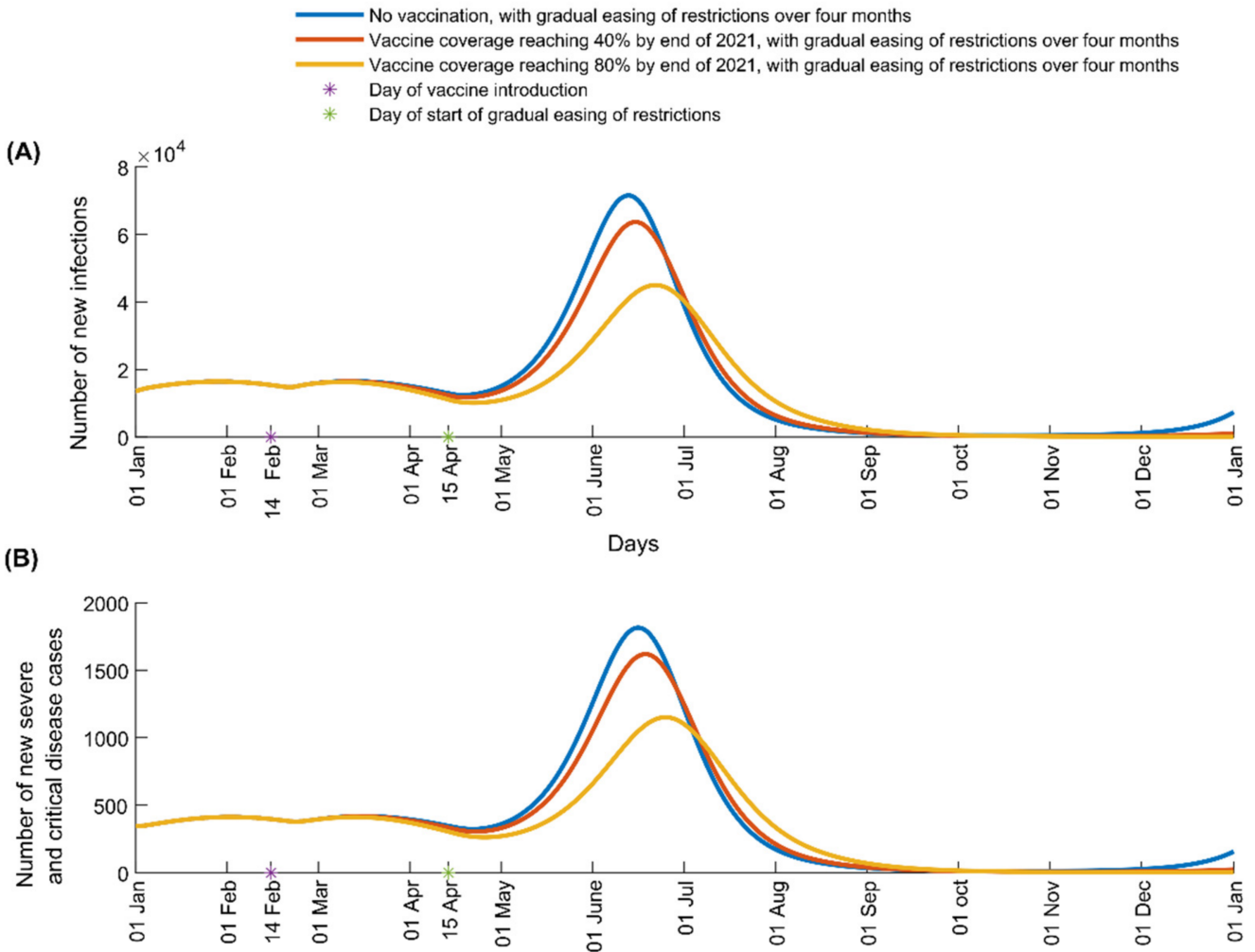

(C)

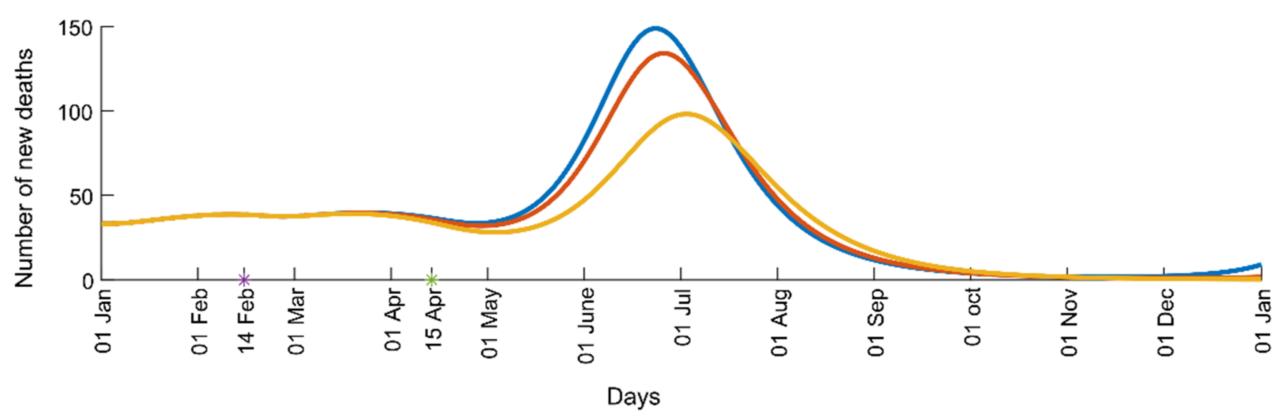

Figure 1. Impact of SARS-CoV-2 vaccination on number of (A) new infections, (B) new severe and critical disease cases, and (C) new deaths in Lebanon. The vaccine is introduced on 14 February 2021 and vaccine coverage is scaled up to reach $40 \%$ (red curve) or $80 \%$ (yellow curve) by 31 December 2021. The simulations assume $R_{0}$ of 1.2 from 1 January 2021 to 15 April 2021 when it starts to increase with gradual easing of restrictions to reach 6.0 after four months. 

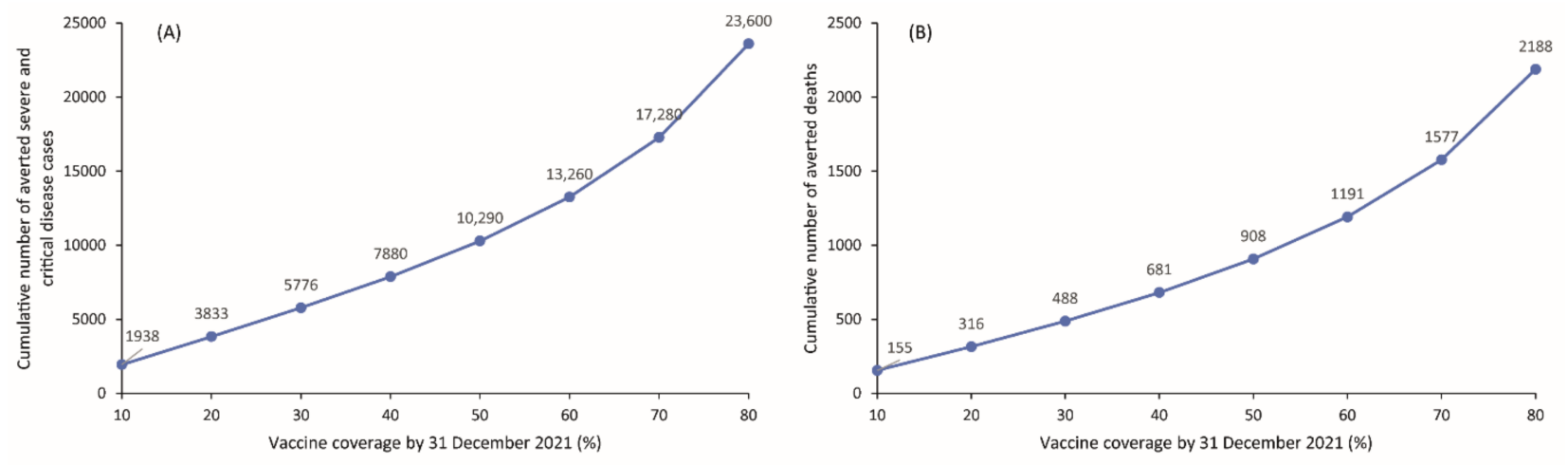

Figure 2. Impact of various levels of vaccine coverage reached by 31 December 2021 on the cumulative number of averted (A) severe and critical disease cases and (B) deaths from 14 February 2021 (date of vaccine introduction) to 31 December 2021. The scenarios assume gradual easing of all restrictions over four months starting 15 April 2021.
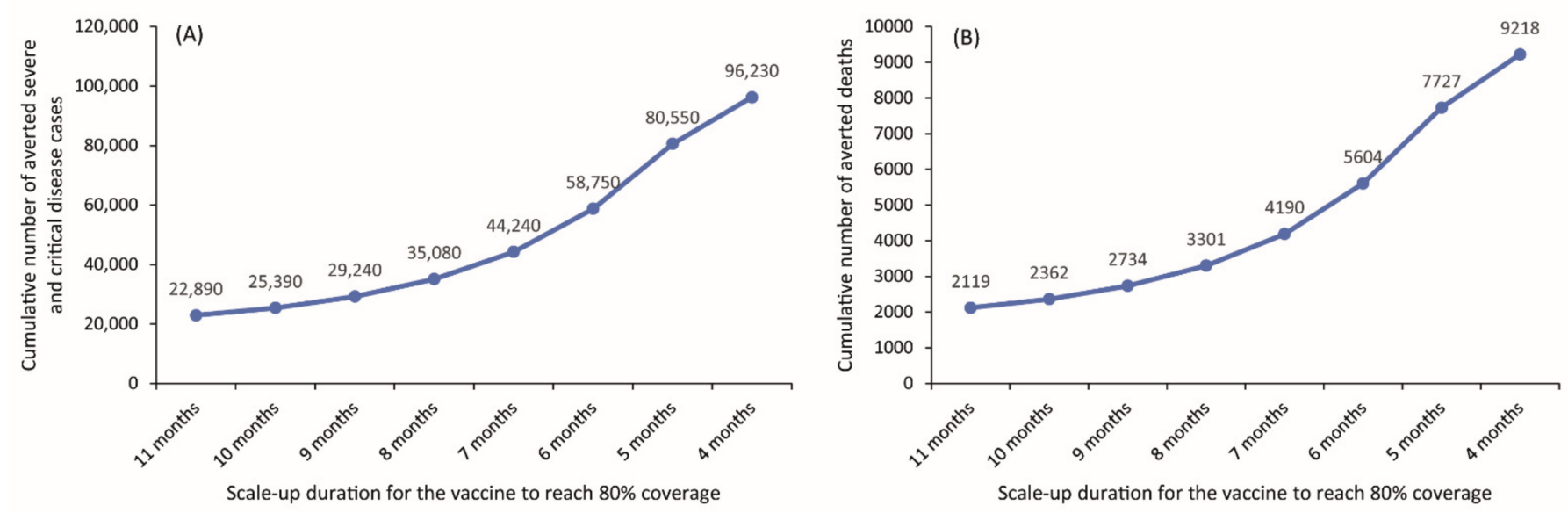

Figure 3. Impact of vaccine scale-up duration to reach $80 \%$ coverage by 31 December 2021 on the cumulative number of averted (A) severe and critical disease cases and (B) deaths from 14 February 2021 (date of vaccine introduction) to 31 December 2021. The scenarios assume gradual easing of all restrictions over four months starting 15 April 2021.
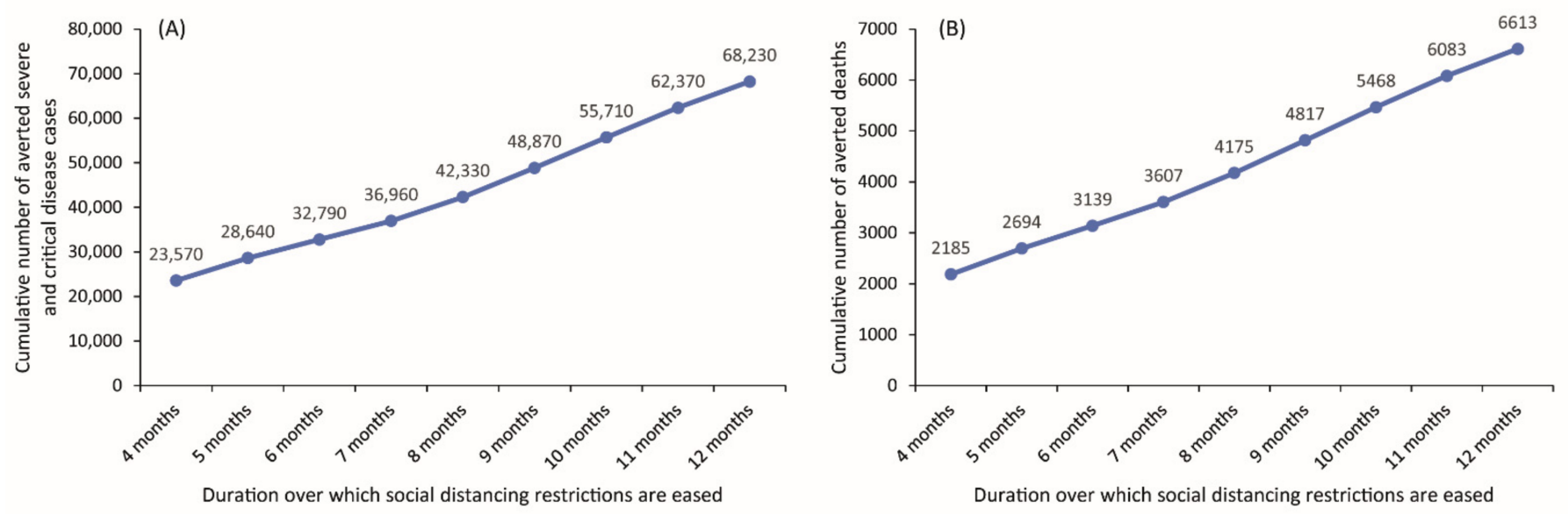

Figure 4. Impact of the duration of easing social and physical distancing restrictions on the cumulative number of averted (A) severe and critical disease cases and (B) deaths from 14 February 2021 (date of vaccine introduction) to 31 December 2021. The scenarios assume gradual easing of all restrictions over four months starting 15 April 2021 and reaching $80 \%$ vaccine coverage by 31 December 2021. 


\section{Discussion}

Our analysis predicts that current held immunity levels in Lebanon, estimated by the model at $40 \%$ on 15 April 2021, are still not sufficient to go back to normalcy and will result in a large new epidemic surge if more transmissible variants of concern are introduced and if restrictions are eased. We found that for vaccination to make an impact on this predicted surge, its scale-up has to be rapid; otherwise, a large fraction of people in the community will be infected before they get vaccinated. For substantial impact, high levels of vaccine coverage also need to be achieved, as the gains from vaccination did not increase linearly with coverage and were most pronounced starting at $70 \%$ coverage. While beneficial, intermediate coverage will not be sufficient to allow return of social contacts to normalcy in the near term and will not spare substantial COVID-19 hospitalizations and deaths. Reaching, for example, $40 \%$ coverage by the end of the year-which is the more likely scenario in Lebanon given current vaccination pace-means a roll-out that is too slow against fast moving incidence, resulting in a more limited impact of vaccination. Our analyses also highlight the importance of keeping social distancing restrictions as long as possible during vaccine scale-up to maximize the benefits of vaccination.

Previous modeling work has shown that vaccine impact is highly dependent on the time of vaccine introduction, with more favorable outcomes in low incidence settings [18,19]. When the vaccine is introduced in a setting with relatively high incidence (such as the case of Lebanon) the impact of the vaccine will be moderate in the short term, despite the high vaccine effectiveness, because time to infection is shorter than the waiting time to vaccination $[18,19]$. This is why we found that that vaccination will flatten the epidemic curve of a predicted summer surge in Lebanon but will not prevent it or delay it, unless scale-up to reach high vaccination coverage (at least 70\%) is very rapid-for example achieved in August rather than December 2021, while maintaining social distancing restriction during roll-out (Figure S2).

At the pace vaccination is taking place, where only $5.1 \%$ of the population have received two doses of the vaccine in three months, it is unlikely that vaccination will have a noticeable impact on a potential surge in the near future. The likelihood of this surge is high given the circulation worldwide of highly infectious SARS-CoV-2 variants of concern $[13,17,38]$, the poor border control implemented in the country, and absence of systematic viral genome sequencing or multiplex PCR variant screening to track the introduction of variants. Social contacts are also expected to increase over the summer months with the arrival of large numbers of Lebanese expatriates and other tourists. This is further motivated by a false sense of security due to the recent marked decline in the number of confirmed cases, as immunity had been building up mainly because of ongoing incidence [1]. There is, therefore, an urgent need to rapidly scale-up vaccination to avoid a surge that the country, with its multiple crises and limited health-care capacity, is largely unprepared for.

Multiple strategies can be followed to overcome supply, administration, and demand challenges to speed up community immunization in Lebanon. While some of the supply bottlenecks result from the high global demand on manufacturers and the prioritization of high-income countries for delivery of reserved doses due to early agreements [39], the government may adopt simple strategies to maximize the use of available doses. The recent decision by the Lebanese authorities to delay administration of the second dose is one good example [40]. Promoting the WHO-approved extraction of one additional jab per vial of the BNT162b2 vaccine $[41,42]$ and reinforcing prioritization of available doses for individuals at higher risk of developing severe disease $[18,35]$ are other suggestions that would capitalize on current supply and maximize its impact. Efforts also need to be made to expand the vaccine distribution campaign to cover all geographical and remote areas, and reach out to the most underprivileged communities. Currently, vaccination is being deployed only through major hospitals. Expanding vaccination sites to include primary health care centers and other existing health care infrastructures, as well as specifically establishing sites such as drive-through vaccination facilities or vaccination centers in sports arenas, could 
be considered. Particular consideration needs to be made to reach the most vulnerable, including refugees, migrant workers, and other underprivileged communities who may not have access to the digital registration platform or to vaccination sites. Mobile vaccination units, partnering with municipalities, and engaging local community organizations would help enhance equitable distribution of the vaccine. Finally, the vaccination campaign should be accompanied by a strategic communication plan, clear and tailored awareness campaigns, and community engagement initiatives to address vaccine hesitancy and increasing the public's confidence in vaccines and in the national vaccination program.

This study has some limitations. Model estimations are contingent on the validity and generalizability of input data. While current available natural history and epidemiological evidence was used to justify model assumptions and parameter values, our understanding of this infection is still evolving. We assumed a reproduction number of six following easing of restrictions to account for the increased transmissibility of the variants of concerns, but this value may differ for different lineages. Still, we found that uncertainty around this parameter does not affect the beneficial vaccine impact relative to the no vaccination scenario (Figure S1). Data on SARS-CoV-2 seroprevalence in the Lebanese population are lacking. The level of prior exposure to the infection plays an important role in this analysis and will affect estimates of vaccine impact. We assumed that $20 \%$ had been exposed to the infection on 1 January 2021, a sensible estimate based on triangulation of available local data, including the cumulative number of confirmed infections and deaths and routine clinical antibody testing data. We assumed that both natural and vaccineinduced immunity last for one year, as suggested by available data [21-24]. However, these two important parameters remain unknown. If they prove to be less than the assumed one year, the impact of the vaccine will be reduced. The model did not prioritize vaccination based on age, since younger individuals in the workforce are currently being vaccinated through the private sector. Given that the highest risk elderly population ( $>70$ years) did receive the vaccine first, our estimates for the impact of vaccination are conservative and the number of averted disease cases and deaths may be higher than the ones reported in our study. Finally, analyses were conducted at a national level, whereas vaccine impact will likely differ between geographic locations with different epidemic transmission dynamics, different levels of compliance to preventive measures, and different vaccine uptake levels.

\section{Conclusions}

In conclusion, amidst all ongoing crises in the country, Lebanon cannot afford the burden of yet another large epidemic wave. Given the dire economic situation, strict lockdowns and extended closures may also be difficult to sustain. At this stage, scaling up vaccination offers the only hope to go back to normalcy with the least deaths and burden on the health care system possible, and with the least damage to the economy in the short and longer term. Our findings reiterate that there is every virtue in speeding up vaccine roll-out and increasing coverage to avoid the potentially devastating consequences of a predicted and likely epidemic surge. With only 6.8 million inhabitants, this goal should be feasible. However, concerted efforts need to be put to overcome local challenges. In the meantime, public messages need to promote adherence to social distancing measures until we approach high levels of vaccine coverage.

Supplementary Materials: The following are available online at https:/ / www.mdpi.com/article/10 .3390 /vaccines9070697/s1, Text S1A: Model structure, Text S1B: Model equations, Text S1C: Model parameters, Table S1: Definitions of population variables and symbols used in the model, Table S2: Model assumptions in terms of parameter values, Figure S1: Schematic diagram describing the SARS-CoV-2 transmission dynamics model in presence of a vaccine that reduces susceptibility to infection, Figure S2: Analysis of the effect of uncertainty around the transmissibility (R0) of the new variants introduced on 15 April 2021, Figure S3: Impact of SARS-CoV-2 vaccination on number of (A) new infections, (B) new severe and critical disease cases, and (C) new deaths in Lebanon for vaccine coverage of $80 \%$ by 31 August 2021. 
Author Contributions: Conceptualization, G.R.M., F.E.-J., L.J.A.-R.; methodology, G.R.M., L.J.A.-R., M.M.; validation, G.R.M., L.J.A.-R., M.M.; formal analysis, M.M.; software, M.M.; writing-original draft preparation, G.R.M.; writing-review and editing, G.R.M., F.E.-J., M.M., M.J., A.H., L.J.A.-R.; visualization, G.R.M., M.M.; supervision, G.R.M., F.E.-J., L.J.A.-R., M.M.; funding acquisition, G.R.M., L.J.A.-R. All authors have read and agreed to the published version of the manuscript.

Funding: This paper was supported with funding from the Global Challenges Research Fund (GCRF) through UKRI/ESRC for the 'RECAP' project (ES/P010873/1). The developed mathematical models were made possible by NPRP grant number 9-040-3-008 (Principal investigator: LJA) and NPRP grant number 12S-0216-190094 (Principal investigator: LJA) from the Qatar National Research Fund (a member of Qatar Foundation; https: / / www.qnrf.org, accessed on 22 June 2021). The statements made herein are solely the responsibility of the authors.

Institutional Review Board Statement: Not applicable.

Informed Consent Statement: Not applicable.

Data Availability Statement: All data are available in the manuscript and its supplementary materials.

Acknowledgments: The authors are grateful for support provided by the Biomedical Research Program and the Biostatistics, Epidemiology, and Biomathematics Research Core, both at Weill Cornell Medicine-Qatar.

Conflicts of Interest: The authors declare no conflict of interest. The funders had no role in the design of the study; in the collection, analyses, or interpretation of data; in the writing of the manuscript, or in the decision to publish the results.

\section{References}

1. Lebanon COVID-19 Dashboard. Key Indicators Based on Publicly Available Information. Available online: https://infogram. com/lebanon-covid-19-dashboard-1h7g6k3z3jpo2oy?live (accessed on 22 April 2021).

2. United Nations Department of Economic and Social Affairs Population Dynamics. The 2019 Revision of World Population Prospects. Available online: https:/ / population.un.org/wpp/ (accessed on 29 April 2021).

3. UNHCR. Syria Regional Refugee Response-4 November 2020. Available online: https://data2.unhcr.org/en/situations/syria\# ga=2.115689286.1120363714.1587065689-968033415.1586509661 (accessed on 18 November 2020).

4. Fouad, F.M.; McCall, S.J.; Ayoub, H.; Abu-Raddad, L.J.; Mumtaz, G.R. Vulnerability of Syrian refugees in Lebanon to COVID-19: Quantitative insights. Confl. Health 2021, 15, 13. [CrossRef] [PubMed]

5. Polack, F.P.; Thomas, S.J.; Kitchin, N.; Absalon, J.; Gurtman, A.; Lockhart, S.; Perez, J.L.; Pérez Marc, G.; Moreira, E.D.; Zerbini, C.; et al. Safety and Efficacy of the BNT162b2 mRNA Covid-19 Vaccine. N. Engl. J. Med. 2020, 383, 2603-2615. [CrossRef] [PubMed]

6. Voysey, M.; Clemens, S.A.C.; Madhi, S.A.; Weckx, L.Y.; Folegatti, P.M.; Aley, P.K.; Angus, B.; Baillie, V.L.; Barnabas, S.L.; Bhorat, Q.E.; et al. Safety and efficacy of the ChAdOx1 nCoV-19 vaccine (AZD1222) against SARS-CoV-2: An interim analysis of four randomised controlled trials in Brazil, South Africa, and the UK. Lancet 2021, 397, 99-111. [CrossRef]

7. Baden, L.R.; El Sahly, H.M.; Essink, B.; Kotloff, K.; Frey, S.; Novak, R.; Diemert, D.; Spector, S.A.; Rouphael, N.; Creech, C.B.; et al. Efficacy and Safety of the mRNA-1273 SARS-CoV-2 Vaccine. N. Engl. J. Med. 2020, 384, 403-416. [CrossRef]

8. U.S. Food and Drug Administration. Janssen COVID-19 Vaccine Frequently Asked Questions. Available online: https: //www.fda.gov/emergency-preparedness-and-response/mcm-legal-regulatory-and-policy-framework/janssen-covid-19 -vaccine-frequently-asked-questions\#:\%7E:text=United $\% 20$ States $\% 3 \mathrm{~A} \% 20$ the $\% 20$ vaccine $\% 20$ was, 28 $\% 20$ days $\% 20$ after $\% 20$ vaccination \%2C\%20respectively (accessed on 22 June 2021).

9. Logunov, D.Y.; Dolzhikova, I.V.; Shcheblyakov, D.V.; Tukhvatulin, A.I.; Zubkova, O.V.; Dzharullaeva, A.S.; Kovyrshina, A.V.; Lubenets, N.L.; Grousova, D.M.; Erokhova, A.S.; et al. Safety and efficacy of an rAd26 and rAd5 vector-based heterologous prime-boost COVID-19 vaccine: An interim analysis of a randomised controlled phase 3 trial in Russia. Lancet 2021, 397, 671-681. [CrossRef]

10. Amit, S.; Regev-Yochay, G.; Afek, A.; Kreiss, Y.; Leshem, E. Early rate reductions of SARS-CoV-2 infection and COVID-19 in BNT162b2 vaccine recipients. Lancet 2021, 397, 875-877. [CrossRef]

11. Tande, A.J.; Pollock, B.D.; Shah, N.D.; Farrugia, G.; Virk, A.; Swift, M.; Breeher, L.; Binnicker, M.; Berbari, E.F. Impact of the COVID-19 Vaccine on Asymptomatic Infection Among Patients Undergoing Pre-Procedural COVID-19 Molecular Screening. Clin. Infect Dis. 2021. [CrossRef]

12. Dagan, N.; Barda, N.; Kepten, E.; Miron, O.; Perchik, S.; Katz, M.A.; Hernan, M.A.; Lipsitch, M.; Reis, B.; Balicer, R.D. BNT162b2 mRNA Covid-19 Vaccine in a Nationwide Mass Vaccination Setting. N. Engl. J. Med. 2021, 384, 1412-1423. [CrossRef]

13. Abu-Raddad, L.J.; Chemaitelly, H.; Butt, A.A.; National Study Group for, C.-V. Effectiveness of the BNT162b2 Covid-19 Vaccine against the B.1.1.7 and B.1.351 Variants. N. Engl. J. Med. 2021. [CrossRef]

14. Inter-Ministerial and Municipal Platform for Assessment, C.a.T.I. Available online: https://impact.cib.gov.lb/home? dashboardName=vaccine (accessed on 29 April 2021). 
15. Davies, N.G.; Abbott, S.; Barnard, R.C.; Jarvis, C.I.; Kucharski, A.J.; Munday, J.D.; Pearson, C.A.B.; Russell, T.W.; Tully, D.C.; Washburne, A.D.; et al. Estimated transmissibility and impact of SARS-CoV-2 lineage B.1.1.7 in England. Science 2021. [CrossRef]

16. Challen, R.; Brooks-Pollock, E.; Read, J.M.; Dyson, L.; Tsaneva-Atanasova, K.; Danon, L. Risk of mortality in patients infected with SARS-CoV-2 variant of concern 202012/1: Matched cohort study. BMJ 2021, 372, n579. [CrossRef]

17. Abdool Karim, S.S.; de Oliveira, T. New SARS-CoV-2 Variants-Clinical, Public Health, and Vaccine Implications. N. Engl. J. Med. 2021. [CrossRef]

18. Makhoul, M.; Ayoub, H.H.; Chemaitelly, H.; Seedat, S.; Mumtaz, G.R.; Al-Omari, S.; Abu-Raddad, L.J. Epidemiological Impact of SARS-CoV-2 Vaccination: Mathematical Modeling Analyses. Vaccines 2020, 8, 668. [CrossRef]

19. Makhoul, M.; Chemaitelly, H.; Ayoub, H.H.; Seedat, S.; Abu-Raddad, L.J. Epidemiological Differences in the Impact of COVID-19 Vaccination in the United States and China. Vaccines 2021, 9, 223. [CrossRef]

20. Ayoub, H.H.; Chemaitelly, H.; Makhoul, M.; Kanaani, Z.A.; Kuwari, E.A.; Butt, A.A.; Coyle, P.; Jeremijenko, A.; Kaleeckal, A.H.; Latif, A.N.; et al. Epidemiological impact of prioritising SARS-CoV-2 vaccination by antibody status: Mathematical modelling analyses. BMJ Innov. 2021, 7, 327-336. [CrossRef]

21. Abu-Raddad, L.J.; Chemaitelly, H.; Malek, J.A.; Ahmed, A.A.; Mohamoud, Y.A.; Younuskunju, S.; Ayoub, H.H.; Al Kanaani, Z.; $\mathrm{Al}$ Khal, A.; Al Kuwari, E.; et al. Assessment of the risk of SARS-CoV-2 reinfection in an intense re-exposure setting. Clin. Infect Dis. 2020. [CrossRef]

22. Wajnberg, A.; Amanat, F.; Firpo, A.; Altman, D.R.; Bailey, M.J.; Mansour, M.; McMahon, M.; Meade, P.; Mendu, D.R.; Muellers, K.; et al. Robust neutralizing antibodies to SARS-CoV-2 infection persist for months. Science 2020, 370, 1227-1230. [CrossRef]

23. Wajnberg, A.; Mansour, M.; Leven, E.; Bouvier, N.M.; Patel, G.; Firpo-Betancourt, A.; Mendu, R.; Jhang, J.; Arinsburg, S.; Gitman, M.; et al. Humoral response and PCR positivity in patients with COVID-19 in the New York City region, USA: An observational study. Lancet Microbe 2020, 1, e283-e289. [CrossRef]

24. Dan, J.M.; Mateus, J.; Kato, Y.; Hastie, K.M.; Yu, E.D.; Faliti, C.E.; Grifoni, A.; Ramirez, S.I.; Haupt, S.; Frazier, A.; et al. Immunological memory to SARS-CoV-2 assessed for up to 8 months after infection. Science 2021, 371. [CrossRef]

25. Abu-Raddad, L.J.; Chemaitelly, H.; Coyle, P.; Malek, J.A.; Ahmed, A.A.; Mohamoud, Y.A.; Younuskunju, S.; Ayoub, H.H.; Al Kanaani, Z.; Al Kuwari, E.; et al. SARS-CoV-2 antibody-positivity protects against reinfection for at least seven months with $95 \%$ efficacy. EClinicalMedicine 2021, 35, 100861. [CrossRef]

26. The Language of Technical Computing; The MathWorks, Inc.: Natick, MA, USA, 2019.

27. He, W.; Yi, G.Y.; Zhu, Y. Estimation of the basic reproduction number, average incubation time, asymptomatic infection rate, and case fatality rate for COVID-19: Meta-analysis and sensitivity analysis. J. Med. Virol. 2020. [CrossRef]

28. MIDAS Online COVID-19 Portal. COVID-19 Parameter Estimates: Basic Reproduction Number. Available online: https:/ /github. com/midas-network/COVID-19/tree/master/parameter_estimates/2019_novel_coronavirus (accessed on 19 May 2020).

29. Korber, B.; Fischer, W.M.; Gnanakaran, S.; Yoon, H.; Theiler, J.; Abfalterer, W.; Hengartner, N.; Giorgi, E.E.; Bhattacharya, T.; Foley, B.; et al. Tracking Changes in SARS-CoV-2 Spike: Evidence that D614G Increases Infectivity of the COVID-19 Virus. Cell 2020, 182, 812-827.e819. [CrossRef]

30. Grubaugh, N.D.; Hanage, W.P.; Rasmussen, A.L. Making Sense of Mutation: What D614G Means for the COVID-19 Pandemic Remains Unclear. Cell 2020, 182, 794-795. [CrossRef]

31. Lauring, A.S.; Hodcroft, E.B. Genetic Variants of SARS-CoV-2-What Do They Mean? JAMA J. Am. Med. Assoc. 2021. [CrossRef]

32. Hall, V.J.; Foulkes, S.; Saei, A.; Andrews, N.; Oguti, B.; Charlett, A.; Wellington, E.; Stowe, J.; Gillson, N.; Atti, A.; et al. COVID-19 vaccine coverage in health-care workers in England and effectiveness of BNT162b2 mRNA vaccine against infection (SIREN): A prospective, multicentre, cohort study. Lancet 2021. [CrossRef]

33. World Health Organization. Clinical Management of COVID-19. Available online: https://www.who.int/publications-detail/ clinical-management-of-covid-19 (accessed on 31 May 2020).

34. World Health Organization. International Guidelines for Certification and Classification (Coding) of COVID-19 as Cause of Death. Available online: https://www.who.int/classifications/icd/Guidelines_Cause_of_Death_COVID-19-20200420-EN.pdf? ua=1.DocumentNumber:WHO/HQ/DDI/DNA/CAT (accessed on 1 June 2020).

35. Bubar, K.M.; Reinholt, K.; Kissler, S.M.; Lipsitch, M.; Cobey, S.; Grad, Y.H.; Larremore, D.B. Model-informed COVID-19 vaccine prioritization strategies by age and serostatus. Science 2021. [CrossRef] [PubMed]

36. Mckay, M.D.; Beckman, R.J.; Conover, W.J. A Comparison of Three Methods for Selecting Values of Input Variables in the Analysis of Output from a Computer Code. Technometrics 1979, 21, 239-245. [CrossRef]

37. Sanchez, M.A.; Blower, S.M. Uncertainty and sensitivity analysis of the basic reproductive rate-Tuberculosis as an example. Am. J. Epidemiol. 1997, 145, 1127-1137. [CrossRef]

38. India's COVID-19 emergency. Lancet 2021, 397, 1683. [CrossRef]

39. Wouters, O.J.; Shadlen, K.C.; Salcher-Konrad, M.; Pollard, A.J.; Larson, H.J.; Teerawattananon, Y.; Jit, M. Challenges in ensuring global access to COVID-19 vaccines: Production, affordability, allocation, and deployment. Lancet 2021, 397, 1023-1034. [CrossRef]

40. Plotkin, S.A.; Halsey, N. Accelerate COVID-19 Vaccine Rollout by Delaying the Second Dose of mRNA Vaccines. Clin. Infect. Dis. 2021. [CrossRef]

41. European Centre for Disease Prevention and Control. Overview of the Implementation of COVID-19 Vaccination Strategies and Vaccine Deployment Plans in the EU/EEA—EN; European Centre for Disease Prevention and Control: Stockholm, Sweden, 2021.

42. WHO. Why Are There Extra Doses of Vaccine in the Vaccine Vial? WHO: Geneva, Switzerland, 2021. 